\title{
On the mechanism of anti-CD39 immune checkpoint therapy
}

\author{
David Allard, ${ }^{1}$ Bertrand Allard, ${ }^{2}$ John Stagg ${ }^{1,2}$
}

To cite: Allard D, Allard B, Stagg J. On the mechanism of anti-CD39 immune checkpoint therapy. Journal for ImmunoTherapy of Cancer 2020;8:e00186. doi:10.1136/ jitc-2019-000186

- Additional material is published online only. To view please visit the journal online (http://dx.doi.org/10.1136/jitc2019-000186).

Accepted 28 December 2019

Check for updates

(C) Author(s) (or their employer(s)) 2020. Re-use permitted under CC BY-NC. No commercial re-use. See rights and permissions. Published by BMJ.

${ }^{1}$ Faculty of Pharmacy, Centre Hospitalier de L'Universite de Montreal, Montreal, Quebec, Canada

${ }^{2}$ Institut du Cancer de

Montreal, Centre Hospitalier de L'Universite de Montreal, Montreal, Quebec, Canada

Correspondence to

Dr John Stagg;

john.stagg@umontreal.ca

\begin{abstract}
With the coming of age of cancer immunotherapy, the search for new therapeutic targets has led to the identification of immunosuppressive adenosine as an important regulator of antitumor immunity. This resulted in the development of selective inhibitors targeting various components of the adenosinergic pathway, including small molecules antagonists targeting the high affinity A2A adenosine receptor and low affinity A2B receptor, therapeutic monoclonal antibodies (mAbs) and small molecules targeting CD73 and therapeutic mAbs targeting CD39. As each regulator of the adenosinergic pathway present non-overlapping biologic functions, a better understanding of the mechanisms of action of each targeted approach should accelerate clinical translation and improve rational design of combination treatments. In this review, we discuss the potential mechanisms-ofaction of anti-CD39 cancer therapy and potential toxicities that may emerge from sustained CD39 inhibition. Caution should be taken, however, in extrapolating data from gene-targeted mice to patients treated with blocking antiCD39 agents. As phase I clinical trials are now underway, further insights into the mechanism of action and potential adverse events associated with anti-CD39 therapy are anticipated in coming years.
\end{abstract}

\section{INTRODUCTION}

The development of adenosine-targeting agents stems from foundational insights from a large body of biochemical, immunological, genetic and pharmacologic studies demonstrating the broad immunosuppressive effects of extracellular adenosine ${ }^{1-3}$ Notably, using mice genetically deficient in adenosine A2A receptor, Sitkovsky and colleagues provided the first genetic evidence of the critical importance of the adenosinergic pathway in tumor immunity.

\section{CD39 and ATP signaling}

ATP is released by dying or stressed cells in order to provide inflammatory signals crucial for effective innate and adaptive immune responses. Conversely, hydrolysis of extracellular ATP into adenosine serves to limit immune responses. CD39 (ecto-nucleoside triphosphate diphosphohydrolase-1 (ENTPD1)) is the rate-limiting ecto-enzyme in the hydrolysis of extracellular adenosine triphosphate (ATP).

CD39 and adenosine receptors are upregulated in response to various stimuli such as hypoxia, tissue damage and remodeling (eg, hypoxia-induced factor (HIF)-1, transforming growth factor (TGF) $\beta$ and epithelial-to-mesenchymal-transition) and in response to chronic inflammation (eg, interleukin (IL)-6, tumor necrosis factor-alpha (TNF- $\alpha$ ) and $\mathrm{T}$ cell exhaustion). CD39 has further been reported to be upregulated by IL-27, oxidative stress and aryl hydrocarbon receptor (AHR). ${ }^{5}$

Through enhanced expression of CD39, tumors are thus proficient at depleting immunostimulatory ATP. ${ }^{6}$ High levels of CD39 have been reported in various human tumors, including in pancreatic cancer, ovarian cancer, lung cancer, kidney cancer, thyroid cancer, testicular cancer, lymphoma, sarcoma and chronic lymphocytic leukemia. ${ }^{7-10}$ While in some cases, tumor cells can overexpress CD39 compared with normal cells, ${ }^{7}$ cell types most frequently expressing CD39 in the tumor microenvironment (TME) are fibroblasts, myeloid cells, vascular endothelial cell, $\mathrm{T}$ regulatory cells (Tregs) and tumor-specific $\mathrm{T}$ effector cells. CD73 is also prevalent in human tumors, and cell types most frequently expressing CD73 are tumor cells, myeloid cells, fibroblasts and endothelial cells. ${ }^{6}$

Present in negligible concentrations under normal conditions (10-100 nM), extracellular ATP rapidly increases in response to tissue injury and hypoxia ${ }^{11}$ and can be found at high concentrations in tumors $(1-50 \mu \mathrm{M}){ }^{12}$ ATP can be released from various cell types, either passively as a result of cellular necrosis or in a controlled manner most often through specialized channels such as pannexins (eg, Panx1) or hemichannel connexins (eg, Conx37 and Conx44). Once released, extracellular ATP signals through type 2 purinergic (P2) receptors, consisting of eight P2Y G-protein coupled receptors (GPCRs) and seven P2X cationselective channel receptors (P2XR; although only P2X1 P2X2, P2X3, P2X4, and P2X7 are 
believed to be functional). Notably, all P2X receptors can assemble into heteromeric receptors, with the exception of $\mathrm{P} 2 \mathrm{X} 7$, which is only present as a homo-trimeric receptor. ${ }^{13}$

P2X7 is the most involved ATP receptor in inflammation. It is highly expressed on macrophages, dendritic cells (DCs), granulocytes, $\mathrm{T}$ cells (particularly Tregs) and B cells. In contrast, $\mathrm{P} 2 \mathrm{X} 1$ and $\mathrm{P} 2 \mathrm{X} 4$ are restricted to lymphocytes and macrophages, while P2X2 and P2X3 are restricted to B cells. ${ }^{14}$ While $\mathrm{P} 2 \mathrm{X}$ receptors are selective for ATP, P2Y receptors can also be activated by UTP, ADP, $\mathrm{NADP} / \mathrm{NAD}^{+}$and UDP. In immune cells, activation of $\mathrm{P} \mathrm{Y}^{15}{ }^{15}$ and possibly P2Y6 ${ }^{16}$ enhances myeloid cell chemotaxis, activation of $\mathrm{P} 2 \mathrm{Y} 12$ promotes antigen presentation by DCs in mice, ${ }^{17}$ and activation of P2Y11 (absent in mice) favors M1-like macrophage polarization, ${ }^{18}$ regulates IL-12 and IL-10 release and enhances chemotaxis. Overt P2Y11 stimulation, however, impairs T cell migration and activation by antigen-presenting cells (APCs). ${ }^{19}$

\section{CD39 and adenosine signaling}

By catabolizing the conversion of extracellular ATP into AMP, CD39 also increases extracellular adenosine production via $\mathrm{CD} 73$ (ecto-5'-nucleotidase), the ratelimiting ecto-enzyme in extracellular AMP hydrolysis. Adenosine signals through type 1 purinergic receptors and has opposing effects to those mediated by ATP receptors. All four adenosine receptors (ie, A1, A2A, A2B and A3) are GPCR. A1 and A3 receptors are preferentially coupled to $G_{i / o}$ proteins, inhibiting adenylate cyclase and cyclic AMP production, while A2A and A2B receptors are generally $\mathrm{G}_{\mathrm{s}}$-coupled and trigger intracellular cAMP accumulation. In human, A1, A2A and A3 display high affinity for adenosine (Ki: $100-300 \mathrm{nM}$ ), while A2B receptor has significantly lower affinity (Ki: $10-15 \mathrm{uM}$ ). Both A2A and A2B contribute to adenosine-mediated immunosuppression.

In addition to ecto-nucleotidases, the adenosinergic pathway is regulated by membrane channels (ATP release), concentrative and equilibrative nucleotides transporters, adenosine deaminase and by specific ATP and adenosine receptors with various expression levels and coexpression patterns. Importantly, each regulator of the ATP-adenosinergic pathway has non-overlapping biologic function, evidenced by the different phenotypes of gene-deficient mice. This implies that targeting a specific enzyme, receptor or transporter of the adenosine pathway will likely trigger distinct biologic effects and that combined inhibition of multiple component may be synergistic, as was suggested in preclinical animal studies. ${ }^{20}$

\section{Adenosine signaling in tumor immunity}

Adenosine-mediated immunosuppression has been extensively reviewed elsewhere. ${ }^{6}{ }^{21}$ High affinity A2A and low affinity A2B adenosine receptors induce cyclic AMP response element dependent redirection of transcription in $\mathrm{T}$ cells and myeloid cells. ${ }^{22}$ The importance of adenosine signaling in tumor immunity, particularly through A2A receptors, is best exemplified by the fact that mice globally deficient in A2A or CD73 mount increased antitumor $\mathrm{CD}^{+} \mathrm{T}$ cell-mediated and natural killer (NK) cell-mediated responses, ${ }^{4}$ that targeted blockade of A2A receptors or CD73 synergizes with immune checkpoint blockade, ${ }^{23}$ immunogenic chemotherapies, ${ }^{24}$ BRAF and MEK inhibitors, ${ }^{25}$ adoptive cell therapy ${ }^{26}$ or tumortargeted antibodies such as trastuzumab. ${ }^{27}$ Notably, conditional deletion of A2A in myeloid cells or NK cells significantly enhances antitumor immune responses. ${ }^{28}$ However, conditional deletion of A2A in $\mathrm{T}$ cells has been associated with precipitated activation-induced cell death. $^{29}$

\section{CD39, NLRP3 inflammasome and pyroptosis}

Extracellular ATP is a potent activator of the NLRP3 inflammasome, a cytosolic complex that once activated cleaves caspase-1, which then processes prointerleukin-1 $\beta$ (IL-1 $\beta$ ) and pro-IL-18 to their secreted forms. ${ }^{30}$ IL-1R signaling enhances $\mathrm{CD} 8^{+} \mathrm{T}$ cell function by upregulating effector proteins, such as granzyme B, and by promoting antigen-driven proliferation. ${ }^{31}$ Consistent with an important role for NLRP3 inflammasome in promoting antitumor immunity, anti-PD-1 or anti-CTLA-4 $\mathrm{mAb}$ therapy is less effective in Nlrp3 $3^{-/-}$or Casp1/11 $1^{-/-}$ mice. ${ }^{32}$ Furthermore, inflammasome-related genes are significantly upregulated in anti-PD-1 responder, but not in non-responder, melanoma patients. ${ }^{32}$ These studies support the notion that NLRP3 inflammasome contributes to adaptive antitumor immunity. Yet, NLRP3 inflammasomes and IL-1 signaling can promote tumor growth and have been shown to suppress NK cell function in mice ${ }^{33}$ for instance via IL-1R8 ${ }^{34}$ IL-1 signaling has also been associated with increased chemoresistance in mouse models of cancer ${ }^{35}$ and production of protumorigenic IL-22. ${ }^{36}$ In prognostic studies, higher tumor IL-1 $\beta$ levels have been associated with improved survival of patients with lymph node-positive breast cancer, ${ }^{37}$ while the use of a blocking anti-IL-1 $\beta$ antibody in patients with cardiovascular diseases showed a reduction on lung cancer incidence. $^{38}$

Extracellular ATP activates NLRP3 inflammasome through P2X7 receptors. P2X7 is an ionic channelforming receptor with low affinity for ATP. Thus, only high concentrations of ATP achieved on cellular stress responses, cell death or inflammation can activate P2X7. By increasing $\mathrm{K}^{+}$efflux ${ }^{39}$ P2X7 promotes assembly of NRLP3 complexes and enhances adaptive immunity. This is exemplified in the context of allogeneic hematopoietic stem cell transplantation, where P2X7-deficient recipient mice have shown improved survival, decreased graftversus-host disease, reduced TNF- $\alpha$, IL- 6 and interferon (IFN)- $\gamma$, and increased IL-10 and Tregs. ${ }^{40}$

Human $P 2 R X 7$ is highly polymorphic. At least eight lossof-function single nucleotide polymorphisms (SNPs) have been identified, ${ }^{41}$ the best characterized being E496A polymorphism (rs3751143). ${ }^{42}$ Notably, breast cancer patients with this loss-of-function E496A substitution 
display shorter metastatic disease-free survival, ${ }^{43}$ consistent with a critical role for extracellular ATP in promoting antitumor responses. Three gain-of-function P2RX7 SNPs have been identified: H155Y, H270R, and A348T. ${ }^{44-46}$ Notably, haplotypes containing the A348T polymorphism (rs1718119) present a threefold increase in activity. ${ }^{46}$ Whether polymorphisms in P2RX7 are associated with clinical responses to immune checkpoint blockade remains to be assessed.

ATP-mediated activation of NLPR3 inflammasome is also associated with cell death by pyroptosis. ${ }^{47-49}$ Pyroptosis promotes the clearance of pathogens by removing intracellular replication niches and enhancing the host's defensive responses through the production of proinflammatory cytokines. Importantly, pyroptosis can occur in the absence of IL-1 $\beta$ and IL-18 production. Uncoupling of cytokine release and pyroptosis is regulated by unique functions of caspase- 1 and caspase- $11^{50}$ and by the Toll-like receptor adaptor SARM (Sterile $\alpha$ and HEAT Armadillo motif-containing protein). A recent study demonstrated that SARM expression in macrophages suppresses the association between ASC and NLRP3, thus preventing inflammasome activation but increases mitochondrial depolarization in response to specific NLRP3 ligands, thus promoting pyroptosis. ${ }^{51}$ Whether ATPinduced pyroptosis is regulated by SARM and whether SARM is expressed in tumor-associated macrophages remains unknown. Notwithstanding, CD39 inhibition might induce P2X7-mediated pyroptosis of myeloid cells and increased IL-1 $\beta$ and IL-18 production, as suggested by CD39-deficient mice. ${ }^{52}$

\section{CD39 and tumor antigen presentation}

ATP release from dying tumor cells, together with calreticulin exposure and HMGB1 secretion, are hallmarks of immunogenic cell death (ICD). Immune-stimulating ICD has been extensively shown to promote antitumor immune responses. ${ }^{53}$ The antitumor efficacy of certain types of chemotherapy rely on ICD for optimal activity. During ICD, activation P2XR7 and NLRP3 inflammasome in DCs and production of IL-1 $\beta$ are required to prime antitumor T immunity. ${ }^{43}$ Depletion of ATP by CD39 expression on tumor cells has been shown to completely abrogate the antitumor activity of immunogenic chemotherapy. ${ }^{54}$ Consistent with this, administration of a CD39 inhibitor (ARL67156) was shown to restore ICD triggered by mitoxantrone. From a mechanistic point of view, overexpression of CD39 was shown to prevent ATP-dependent

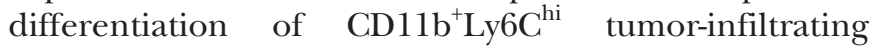
myeloid cells into inflammatory DC-like cells, ultimately impairing antitumor immune responses. ${ }^{55}$

Inhibition of CD39 activity was also shown to restore the sensitivity of autophagy-deficient tumors to immunogenic chemotherapy. Accordingly, blocking CD39 with ARL67156 enhanced tumorous ATP levels and immune control of autophagy-deficient tumors by favoring the recruitment of DCs and IFN- $\gamma$ producing CD4 and CD8 T cells. ${ }^{54}$ Building on this work, Rao et a $\tilde{l}^{6}$ subsequently demonstrated that tumor cell autophagy regulates cancer immunosurveillance through CD39-mediated ATP depletion and activation of adenosine signaling. The authors observed that tumor-specific Atg5 loss induced an HIF $\alpha$-dependent overexpression of CD39 on Kras mutated lung tumor cells. Treatment of KRas;Atg $5^{\mathrm{fl} / \mathrm{fl}}$ mice with a CD39 inhibitor (POM-1) or with a selective A2B receptor antagonist (PSB1115) abolished the accumulation of intratumoral Tregs and reduced the number of tumor foci. This study thus supports the notion that autophagy-deficient tumors can subvert antitumor immunity and favor the early phases of oncogenesis by a mechanism involving Treg recruitment in a CD39 and adenosine-dependent manner.

Further supporting a role for extracellular ATP in promoting antigen presentation, ATP-stimulated DCs upregulate costimulatory molecules and MHC class II, increase their endocytotic activity, produce more IL-12 and show an increased ability to induce $\mathrm{T}$ cell proliferation in vitro. ${ }^{17}{ }^{57}$ Conversely, matured DCs upregulate expression of A2A and A2B receptors that inhibit production of IL-6, IL-12 and IFN- $\gamma^{58}{ }^{58}$ Interestingly, mouse and human liver DCs express higher levels of CD39 than other DCs and are more resistant to prostimulatory effects of exogenous ATP. Consistent with this, CD39-deficient liver DCs exhibit a more mature phenotype, greater responsiveness to TLR ligands and stronger proinflammatory and immunostimulatory activity. ${ }^{59}$ However, chronic stimulation of DCs with extracellular ATP may suppress Th1 responses. Accordingly, chronic ATP exposure was shown to dose-dependently inhibit LPS-dependent and soluble CD40 ligand-dependent production of IL-1, TNF- $\alpha$, IL-6, and IL-12 by DCs. ${ }^{60}$

\section{CD39 and macrophage function}

Mosser and colleagues proposed that 'CD39 acts as a 'molecular switch' that controls the balance between inflammatory and regulatory macrophage differentiation'. ${ }^{61}$ Macrophages secrete, hydrolyze and respond to extracellular ATP. Macrophages further upregulate CD39 in response to $\mathrm{P} 2 \mathrm{X} 7$ activation, thus regulating their function. Notably, blocking CD39 on macrophages significantly enhances their production of TNF- $\alpha$ and IL-12 and decreases their production of IL-10. ${ }^{62} 63$ Consistent with this, CD39-deficient mice exhibit higher levels of inflammatory cytokines in response to LPS-induced sepsis. In contrast, inhibition or deletion of $\mathrm{P} 2 \mathrm{X} 7$ in macrophages attenuates their production of proinflammatory cytokines. Combined P2X7 blockade and A2A receptor activation is required to protect septic CD39-deficient mice against inflammation-induced tissue injury. ${ }^{63}$

Extracellular release of ATP by dead or dying cells also serves as a 'find-me' signal promoting chemotaxis of monocytes, macrophages and neutrophils. ${ }^{1564}$ Notably, ATP is released in a polarized fashion by macrophages, resulting in the accumulation of extracellular ATP near the cell surface closest to the source of chemotactic stimuli in order to amplify chemotactic signals by activating P2Y2 
receptors. However, adding excessive exogenous ATP has been shown to block chemotaxis of macrophages, suggesting that it is the gradient of extracellular ATP concentrations that promote chemotaxis. ${ }^{65}$

Increasing extracellular ATP levels also promotes macrophage phagocytosis. This is mediated through activation of $\mathrm{P} 2 \mathrm{X} 4$ and $\mathrm{P} 2 \mathrm{X} 7$ receptors, which trigger calcium signaling among neighboring macrophages on autocrine and paracrine release of ATP ${ }^{66}$ Thus, ATP signaling promotes communication among macrophages through the propagation of calcium waves to enhance their phagocytic function. Conversely, depletion of ATP by soluble CD39-like apyrase suppressed macrophage phagocytosis in vitro. Notably, 'M1'-like macrophages were more effective in propagating ATP-induced paracrine calcium signaling than 'M2'-like macrophages. Increasing intracellular calcium is known to promote other macrophage function, such as FcR-mediated killing and chemokine signaling. ${ }^{67}$ Whether CD39 expression inhibits FcR signaling remains to be investigated.

Extracellular ATP also stimulates microvesicle release from monocytes and macrophages, a major secretory pathway for rapid production of IL-1 $\beta$ and IL-18. ${ }^{68}$ In macrophages and other myeloid cells, this is mediated by P2X7 activation. In turn, macrophage-derived microvesicles activate unprimed macrophages in a partially TLR4dependent manner. ${ }^{69}$ Macrophages-derived microvesicles serve to induce expression of costimulatory CD86, CD80, CD83, and MHC class II and to increase secretion of TNF- $\alpha$ from other macrophages. A recent study revealed that ATP also redirects TNF trafficking in macrophages, switching off production of the soluble form and increasing the release of transmembrane pro-TNF into microvesicles. ${ }^{70}$ Surprisingly, pro-TNF embedded into these microvesicles appear to be biologically more potent than soluble TNF in vivo. Thus, accumulation of extracellular ATP by targeting CD39 may enhance TNF-mediated responses by redirecting its trafficking to microvesicles.

\section{CD39 and neutrophil function}

In mice, injection of exogenous ATP promotes rapid neutrophil recruitment by a mechanism dependent on caspase-1/11 activation..$^{32}$ Neutrophils also release ATP in response to chemokines. This autocrine signaling serves to amplify chemotactic signals in neutrophils via P2Y2 receptors. ${ }^{65}$ Activation of $\mathrm{P} 2 \mathrm{Y} 2$ receptors on neutrophils has also been shown to promote arachidonic acid release, degranulation and oxidative burst. ${ }^{71}$ Hydrolysis of ATP by CD39-abundant on neutrophils-promotes neutrophil chemotaxis through activation of A3 receptors. In the absence of ATP hydrolysis (by CD39), exogenous ATP has been proposed to promote random neutrophil migration. ${ }^{65}$ In contrast, others have reported increased neutrophil trafficking into the lungs in CD39-deficient mice following LPS-induced challenge ${ }^{72}$ Treating neutrophils with a CD39 inhibitor or siRNA was also shown to increase IL-8 production in response to TLR4 and
TLR1/2 agonists, due to a synergy between TLRs and P2 receptors signaling. ${ }^{71}$

The P2X1 receptor also modulate neutrophil chemotaxis, but in contrast to P2Y2, its serves to stop neutrophil migration once neutrophils reach sites of infection. Following TLR activation with LPS, neutrophils release extracellular ATP to activate P2X1 receptor and stop neutrophil migration. ${ }^{73}$ Notably, adding non-hydrolyzable ATP to neutrophils facilitated the halting of chemotaxis. In contrast, treatment with exogenous hydrolyzable ATP increased neutrophil chemotaxis. Taken together, these studies suggest that blockade of CD39 may promote a stop signal to neutrophil chemotaxis once they reach ATP-rich areas.

\section{CD39 and $\mathrm{T}$ cell function}

Expression of CD39 by activated T cells has been linked to the acquisition of major dysfunctions and exhaustion signature. In support of an important role for CD39 in regulating human T cells, CD39 (ENTPD1) polymorphism associated with reduced CD39 protein expression (A/A of rs10748643) has been associated with increased susceptibility to Crohn's disease ${ }^{74}$ and increased response to vaccination against influenza or live varicella zoster virus. ${ }^{75}$ Individuals carrying the low-expressing CD39 variant display significantly increased vaccine-specific memory $\mathrm{T}$ cell responses. In vitro, $\mathrm{CD} 4^{+} \mathrm{T}$ cell activation in the presence of a CD39 inhibitor (ARL) has been shown to increase IL-2 and IL-21 production, reduce AMPK phosphorylation and restore the ability of CD39 ${ }^{+}$ T cells to help B cell differentiation. ${ }^{75}$ Interestingly, CD39 activity has also been shown to contribute to an increased susceptibility of $\mathrm{CD}^{+} \mathrm{T}$ cells to apoptosis in vitro. In support of this, Fang et $a l^{75}$ found increased induction of CD39 of T cells with age. CD39 ${ }^{+} \mathrm{CD} 4^{+} \mathrm{T}$ cells resembled effector $\mathrm{T}$ cells with signs of metabolic stress and high susceptibility to undergo apoptosis.

Tumor-specific $\mathrm{CD}^{+} \mathrm{T}$ cells found in human tumors express high levels of CD39, are enriched for T cells against neoantigens and exhibit an exhausted gene signature. ${ }^{76}$ Similar findings were reported by three independent groups in human lung cancer, colorectal cancer, breast cancer, ${ }^{77}$ head and neck cancer, ${ }^{78}$ renal cell carcinoma, non-small cell lung cancer and gastric cancer. ${ }^{79}$ $\mathrm{CD} 9^{+} \mathrm{CD}^{+} \mathrm{T}$ cells were found to be enriched within the TME, invaded lymph nodes and metastases compared with healthy tissues, ${ }^{78}{ }^{79}$ peripheral circulation and lymphoid organs. ${ }^{77} \mathrm{CD} 39^{+} \mathrm{CD} 8^{+} \mathrm{T}$ cells are characterized by potent exhaustion featured, including decreased TNF, IL-2 and IFN- $\gamma$ production as well as increased expression of many inhibitory/checkpoint receptors such as PD-1, TIM-3, LAG-3, TIGIT and 2B4. ${ }^{77}$ TCR engagement alone was shown to induced CD39 upregulation on $\mathrm{CD}^{+} \mathrm{T}$ cells in PBMCs of patients with breast cancer. ${ }^{77}$ Several additional factors from the TME might also account for CD39 upregulation in $\mathrm{CD}^{+} \mathrm{T}$ cells, including IL-6 and TGF- $\beta .^{7778}$ Interestingly, expansion of $\mathrm{CD} 39^{+} \mathrm{CD}^{+} \mathrm{T}$ cells 
in blood was found to be associated with clinical responses to anti-PD-1 therapy. ${ }^{76}$

$\mathrm{T}$ cell activation involves ATP release and autocrine stimulation of purinergic $\mathrm{P} 2$ receptors, $\mathrm{P} 2 \mathrm{X} 1, \mathrm{P} 2 \mathrm{X} 4$ and $\mathrm{P} 2 \mathrm{X} 7$, to regulate cellular $\mathrm{Ca}^{2+}$ influx and mitochondrial function, enhancing IL-2 secretion and T cell proliferation. ${ }^{13}$ For instance, localized activation of $\mathrm{P} 2 \mathrm{X} 4$ promotes $\mathrm{Ca}^{2+}$ influx in T cells and selective activation of mitochondria at the immune synapse. In CD4 T cells, stimulation of CXCR4 triggers rapid ATP release that promote T cell migration through autocrine $\mathrm{P} 2 \mathrm{X} 4$ activation. In a mouse lung transplant model, blocking P2X4 with a selective antagonist prevented the recruitment of T cells. ${ }^{80}$

In vitro assays demonstrated that blocking CD39 enhances T cell activation. Accordingly, inhibiting CD39 enzymatic activity significantly enhanced proliferation and function of $\mathrm{CD} 4^{+} \mathrm{T}$ cells and $\mathrm{CD} 8^{+} \mathrm{T}$ cells when cocultured with irradiated SK-MEL-5 human melanoma cells. ${ }^{7}$ Inhibition of CD39 also enhanced cytotoxic function of $\mathrm{CD} 56^{+}$human NK cells in vitro. ${ }^{7}$ Similarly, Häusler $e t a l^{10}$ reported increased antitumor activity of human $\mathrm{CD} 4^{+} \mathrm{T}$ cells against SK-OV-3 and OAW42 ovarian cancer cells.

\section{CD39 and Th17 responses}

Th17 cells are important drivers of chronic inflammation in autoimmune diseases and play an important role in antitumor immunity. Th17 cells are particularly important to regulate autoimmunity and commensal bacteria in the gut. Not surprisingly, extracellular ATP generated by commensal bacteria favors the differentiation of Th17 cells. Activation of P2X7 on monocytes synergizes with TLR ligands to induce a cytokine milieu rich in Th17-promoting IL-23 and IL-1 $\beta$. Notably, P2X7 activation promotes Th17 cell conversion at the expenses of Tregs. Interestingly, CD39 can be expressed on Th17 cells, endowing them with suppressor activity via ATPase activity and associated with increased IL-10 production. In addition to IL-23 and IL-1 $\beta$, although not absolutely required, IL-6, IL-21 and TGF- $\beta$ can also generate Th17 cells. Interestingly, exposure of IL- 6 and TGF- $\beta$ induces STAT3 and downregulates Gfi in a p38-dependent manner, both events being relevant for the induction of CD39 and CD73 by Th17 cells. In patients with cancer, CD39-expressing Th17 cells predict poor clinical outcome. ${ }^{81}$ Th17 cells generated in the absence of TGF- $\beta$ that lack CD39 and CD73 fail to promote tumor growth and are endowed with antitumor functions. ${ }^{82}$

Several studies suggest that Th17 and IL-17A promote antitumor immune responses. For instance, absence of IL-17A-IL-17R signaling reduces tumor-specific $T$ cell responses elicited by ICD. ${ }^{83}$ Agonists of ROR $\gamma$, which promote Th17 responses, have also been shown to enhance antitumor immunity by increasing IL-17A and GM-CSF levels, while diminishing CD39 and CD73 expression ${ }^{84}$ Furthermore, patients with cancer receiving ant-PD-1 checkpoint therapy increase both Th1 and Th17 responses. ${ }^{85}$ In a recent case report, blocking IL-17A was reported to abrogate clinical response to anti-PD-1 therapy. After developing severe psoriatic rash following pembrolizumab treatment, the patient was treated with a blocking anti-IL17A mAb (secukinumab). While blocking IL-17A cured the skin psoriasis, it also abrogated the biochemical response to anti-PD-1 therapy. Together with preclinical studies, this supports an antitumor role for IL-17A during treatment with immune checkpoint inhibitors. $^{86}$

\section{CD39 and NK cell function}

An important role for CD39-mediated immunosuppression of NK cells was recently described. Using metastatic mouse models of melanoma (B16 and LWT1), Zhang et al demonstrated that both CD39 and CD73 are upregulated on lung tumor-infiltrating NK cells. ${ }^{87}$ Strikingly, CD39deficient mice presented reduced experimental lung metastases in both models in which NK cells and IFN- $\gamma$ mediate control of metastasis. ${ }^{87}$ Either NK cell depletion or IFN- $\gamma$ neutralization abrogated the protective effect of CD39 host deficiency in both models. ${ }^{87}$ Targeted blockade of CD39 using POM-1 was further demonstrated to enhance NK cell-mediated metastatic control and synergized with combined Braf and MEK inhibition, recombinant IL-2 or with anti-PD-1 and/or anti-CTLA-4 checkpoint blockade. $^{87}$

\section{CD39 and myeloid-derived suppressor cells (MDSCs)}

Accumulation of MDSC in tumors is recognized as a mean by which cancer to evade antitumor immune responses. Using a cohort of 72 non-small cell lung cancer patients, Li $e t a l^{88}$ reported an accumulation of MDSC expressing both CD39 and CD73 in tumorous tissues of patients with lung cancer. Notably, the authors reported a positive correlation between tumorous ecto-nucleotidasesexpressing MDSC and tumor stage, node involvement and metastasis status. ${ }^{88}$ Expression of CD39 and CD73 on MDSC was shown to be mediated by TGF- $\beta$ and HIF- $1 \alpha$. Pharmacological blockade of CD39 or CD73 on MDSC restored proliferation of cocultured $\mathrm{CD}^{+} \mathrm{T}$ cells as well as survival and IFN- $\gamma$ production of cocultured NK cells. ${ }^{88}$ In vivo, $\mathrm{CD} 39^{+} \mathrm{CD} 73^{+} \mathrm{MDSC}$ abrogated adoptively transferred NK cell-mediated tumor control and chemotherapeutic responses, an effect prevented by treatment with a CD39 inhibitor. ${ }^{88}$ Increased frequencies of $\mathrm{CD} 39^{+} \mathrm{CD} 73^{+}$ MDSC was also reported in colorectal cancer patients. ${ }^{89}$ In this study, granulocytic MDSC expressing high level of PD-L1, CD39, and CD73 displayed enhanced immunosuppressive activity relative to other myeloid cells present in blood. Targeted blockade of CD39 or CD73 could also reverse MDSC-mediated suppressive effects on T cells.

\section{CD39 and immunosuppression through extracellular vesicles (EVs)}

Tumor-derived EVs, which include exosomes, microvesicles and apoptotic bodies, have been shown suppress antitumor T cells including through CD39, CD73 and adenosine signaling. ${ }^{9091}$ Tumor-derived exosomes can also induce CD73 expression on $\mathrm{CD}^{-} 9^{+}$DCs to suppress T cell 
activation. ${ }^{92}$ Various tumor cell types have been reported to generate $\mathrm{CD}^{+} 9^{+} \mathrm{EVs}$, including ovarian cancer, prostate cancer, multiple myeloma, neuroblastoma, and head and neck cancer. ${ }^{92-95}$ More recently, it was shown that B cells also produce high levels of EVs expressing CD39 and CD73, which significantly suppress antitumor immunity, particularly in response to chemotherapy. Notably, administration of B cell-derived EVs abrogated chemotherapymediated tumor-specific $\mathrm{CD} 8^{+} \mathrm{T}$ cells increase in mice, which was prevented by pretreatment of EVs with a CD39 inhibitor (POM-1) or a CD73 inhibitor (APCP). Mechanistically, HIF-1 $\alpha$ activation was shown to promotes EVs production by B cells. Importantly, B cell-derived EVs expressing active CD39 and CD73 were also detected in serum samples of patients with colon cancer or gastric cancer, and high levels of EVs negatively correlated with progression-free survival period in a small cohort. ${ }^{96}$

\section{CD39 and Tregs}

Foxp $3^{+}$Tregs mediate immunosuppression by a number of mechanisms, including via CD39-dependent production of adenosine. ${ }^{97}$ Tregs from $\mathrm{CD} 39^{-/-}$mice display impaired suppressive properties, both in vitro and in vivo. ${ }^{98}$ Conversely, CD39 expression on Tregs ameliorates experimental colitis induced by $\mathrm{T}$ cell transfer. In mice, CD39 ${ }^{+}$Tregs are potent suppressor of Th17 responses and antitumor immunity mediated by NK cells. ${ }^{99}$ Supporting this, the antimetastatic activity of the CD39 inhibitor POM-1 was recently found to be completely abrogated in mice depleted of NK cells or deficient in CD39 expression hematopoietic cells. ${ }^{87}$ CD $39^{+}$Tregs isolated from human colon cancers also suppress proliferation and IFN- $\gamma$ secretion of conventional T cells. ${ }^{100}$ In patients with melanoma, lower baseline levels of circulating $\mathrm{CD} 39^{+} \mathrm{C}-$ $\mathrm{D} 25^{\mathrm{hi}} \mathrm{CD} 4^{+}$Tregs were also found to be significantly associated with better relapse-free survival. ${ }^{101}$

CD39 expression on Tregs is regulated by FoxP3 and TGF- $\beta$. Interestingly, impairment in TGF- $\beta$ signaling in Tregs has been associated with reduced CD39 expression and has been proposed as a mechanism of methotrexate resistance in patients with rheumatoid arthritis (RA). Consistent with an important role for TGF- $\beta$ in regulating CD39 expression, carriers of at least one mutant allele for a TGFBR2 SNP (rs1431131) showed a significant reduction of CD39 mRNA levels in $\mathrm{CD} 4^{+} \mathrm{T}$ cells. ${ }^{102}$

Extracellular ATP and adenosine signaling further regulate Treg survival and function. Notably, P2X7 is highly expressed in Tregs. ${ }^{103}$ Activation of P2X7 by ATP limits Treg-mediated immunosuppression, likely via P2X7-induced cell death. Supporting the notion that anti-CD39 therapy can decrease Treg infiltration in tumors, in vivo treatment with a CD39 antisense oligonucleotide significantly reduced tumor-infiltrating Tregs in mice. ${ }^{104}$ Further in support of a role for extracellular ATP in restricting Treg-mediated suppression, P2X7deficient Tregs show increased suppressive potential and tumor-bearing P2X7null mice present increased tumorinfiltrating Tregs. ${ }^{105}$ A recent study revealed that a large fraction of tumor-infiltrating Tregs actually undergo apoptosis as a consequence of weak NRF2-associated antioxidant system and increased vulnerability to free oxygen species. ${ }^{106}$ Notably, apoptotic Tregs were found to release and convert a large amount of ATP into adenosine, thus suppressing antitumor immunity.

T regulatory type $1(\operatorname{Tr} 1)$ cells represent another subset of regulatory lymphocytes that use CD39 for immunosuppression. Tr1 are characterized by IL-10 production, negativity for Foxp3 and expression of CD49b and LAG-3. Tr1 can be differentiated from CD4 cells on exposure to IL-27 through a mechanism involving AHR and STAT3. Studies have shown that extracellular ATP inhibits Tr1 cell differentiation, while CD39 contributes to Tr1-mediated suppressive activity via adenosine. ${ }^{107} 108$ Since Tr1 cells are induced in the TME, ${ }^{109-111}$ targeting CD39 may also block Tr1-mediated immunosuppression.

\section{CD39 and tumor angiogenesis}

Suppressed tumor growth in CD39-deficient mice has been associated with decreased angiogenesis in several experimental tumor models, including B16F10 melanoma, Lewis lung carcinoma and MC38 colon tumors. ${ }^{99} 112$ Paradoxically, extracellular ATP activates FAK in endothelial cells and promotes their migration, yet activation of FAK is defective in CD39-deficient endothelial cells. This is likely due to desensitization of P2Y2 receptors in endothelial cells on sustained ATP signaling. Since CD39 coexpression with CD73 (in endothelial cells for instance) will ultimately generate adenosine, CD39 also likely promotes angiogenesis via adenosine signaling. Indeed, CD73-mediated adenosine and A2A signaling in endothelial cells have been shown to promote angiogenesis in various experimental conditions, including during tumorigenesis. $^{113}$

\section{CD39 and tumor fibroblasts}

CD39 is highly expressed in tumor-associated fibroblasts in various types of cancers, including ovarian cancer and pancreatic cancer (unpublished observations). CD39 and purinergic P2 receptors are also highly expressed on normal pancreatic stellate cells (PSCs). In a mouse model of chronic pancreatitis and fibrosis, it was shown that CD39-deficient mice develop significantly reduced pancreatic atrophy and limited fibrosis. ${ }^{114}$ Tissue and plasma levels of anti-fibrotic IFN- $\gamma$ were also found to be significantly increased in diseased CD39-deficient mice. Importantly, CD39-deficient PSCs showed significantly decreased rates of proliferation, decreased procollagenalphal production and displayed defective response to mitogenic stimulation by PDGF and to profibrogenic stimulation by TGF- $\beta$. These results suggest a role for CD39 in promoting parenchymal fibrosis in pancreatic tissue. ${ }^{114}$ Similarly, using genetically deficient mice for CD39 and/or CD73, Fernández et al $l^{15}$ demonstrated that lower levels of adenosine in CD39 and/or CD73-deficient mice resulted in diminished dermal fibrosis in a model of scleroderma. The author attributed the mechanism to 
adenosine-mediated excessive collagen production from skin fibroblasts through A2A receptor activation. ${ }^{116}$

Decreased fibrosis observed in $\mathrm{CD}^{-/-}$mice might alternatively result from $\mathrm{P} 2 \mathrm{Y} 2$ receptor desensitization on sustained exposure to high levels of extracellular ATP. In support of this hypothesis, ATP-induced activation of P2Y2 receptor have been shown to enhance IL-6 and collagen type I production by skin fibroblasts, which do not express CD39. ${ }^{117}$ Other studies have also reported profibrotic effects of extracellular ATP. ${ }^{118-121}$

\section{Targeting CD39 in preclinical studies}

Data obtained with CD39-deficent mice strongly support the use of CD39 blocking agents for cancer immunotherapy. Robson and colleagues' seminal work was the first to demonstrate that CD39 knockout mice or mice reconstituted with a CD39-deficent bone marrow were protected against hepatic metastasis of B16F10 melanoma and MCA-38 colon cancer. ${ }^{99}$ Treg-derived CD39 was shown to be critical for tumor growth by suppressing NK cell functions, ${ }^{99}$ while CD39 expression on endothelial cells was proposed to be a main contributor of CD39mediated immunosuppression in the non-hematopoietic compartment. ${ }^{122}$ In accordance with the observations obtained with CD39-deficient animals, enforced overexpression of CD39 in mice promoted metastatic tumor growth in the liver. ${ }^{123}$ Building on this work, the antitumor activity related to pharmacological inhibition of CD39 using small inhibitors or antagonistic monoclonal antibodies has been evaluated in several preclinical cancer models. In monotherapy, CD39 inhibition with POM-1 induces antitumor activity on metastatic tumor growth in models of lung and liver metastasis. ${ }^{87} 99$ Consistent with these findings, targeted blockade of CD39 using an antagonistic monoclonal antibody (clone 9-8B) delayed tumor growth in a PDX model of sarcoma. ${ }^{8}$

Most interestingly, synergistic effects of combination therapy associating CD39 inhibition with immune checkpoint blockade, MAPK inhibitors or immunogenic chemotherapy were recently reported. In lung metastasis models, specific blockade of CD39 with POM-1 significantly enhanced the antitumor activity of anti-PD1 and anti-CTLA-4 mAb, in an NK cell and IFN- $\gamma$ dependent manner. ${ }^{87}$ In another recent study, blocking CD39 with mAb IPH5201 (Innate Pharma) strongly enhanced the antitumor activity of immunogenic chemotherapy in subcutaneous tumor models. ${ }^{124}$ Enhanced activity of antiPD1 and anti-CTLA4 therapy in CD39-deficient mice inoculated with B16 or MCA205 tumors further suggest that targeted blockade of CD39 with IPH5201 might also synergize with immune checkpoint inhibitors. ${ }^{124}$ Mechanistically, IPH5201 helps maintaining extracellular pools of immunogenic ATP by inhibiting both soluble and membrane-bound CD39. Studies in CD39-deficient mice suggested that antitumor activity of IPH5201 resulted from increased tumor infiltration of IFN- $\gamma$-producing T cells. ${ }^{124}$ Similar to the study reported by Innate Pharma, antitumor activity of anti-CD39 mAb, including monotherapeutic activity, against different tumor models were also reported by Tizona Therapeutics and Surface Oncology (Brisbane Immunotherapy 2019 Conference, 23-25 May 2019).

\section{Potential toxicities from CD39 inhibition}

An important phenotype of CD39-deficient mice consists of altered platelet function and increased susceptibility to venous thrombosis. In a recent study, ${ }^{125}$ it was found for instance that when subjected to venous flow restriction, CD39-haploinsufficient mice display significantly more venous thrombosis with increased intravascular fibrin deposition, increased activation of NLRP3 inflammasome and increased IL-1 $\beta$ production. Administration of a blocking against IL-1 $\beta$ antibody rescued increased susceptibility to venous thrombosis, suggesting a CD39-regulated inflammatory mechanism. Thrombi developing in CD39haploinsufficient mice were also significantly more infiltrated with neutrophils, and CD39-deficient neutrophils showed increased formation of neutrophil extracellular trap, which promotes coagulation. While this suggest a potent impact of chronic CD39 inhibition at increasing the risk of thrombosis, administration of anti-CD39 antibodies to mice has not been reported to promote venous thrombosis.

As ADP is a potent activator of platelets (via P2Y12 receptors), depletion of $\mathrm{ADP}$ by CD39 regulates platelet activation and recruitment. Kanthi et al $^{126}$ recently investigated whether this has any impact on atherosclerosis, which is known to be driven in the early stage by platelet activation. By studying CD39 ${ }^{+/-}$; Apoe $^{-/-}$mice on high fat diet, it was found that CD39-haploinsufficiency was associated with larger atherosclerotic lesions attributed to increased platelet activation. Intriguingly, however, total absence of CD39 (ie, CD39-/- mice) did not alter plaque burden, but rather protected mice from atherosclerosis, ${ }^{127}$ possibly because of P2Y receptor desensitization in global CD39-deficient mice. ${ }^{128}$ Administration of anti-CD39 antibodies to mice have not, however, been reported to affect platelet function.

Another potential toxicity might be related to the role of CD39 in the maintenance of liver and pancreas homeostasis. CD39 is the dominant ecto-nucleotidase expressed by hepatic endothelial cells, Kupffer cells, sinusoidal lymphocytes as well as PSCs. Global deletion of CD39 in mice has been associated with metabolic alterations including increased hepatic glucose production, insulin resistance, increased plasma levels of insulin and fatty acids and increased liver inflammation. ${ }^{129}$ Whether antiCD39 targeting agents might affect liver and pancreas function remains to be investigated.

\section{CONCLUSION}

CD39 distributes on a variety of cells in the TME (mostly immune cells, fibroblasts and endothelium) and is upregulated in many human cancers. Because of its critical role as a regulator of both extracellular ATP and 


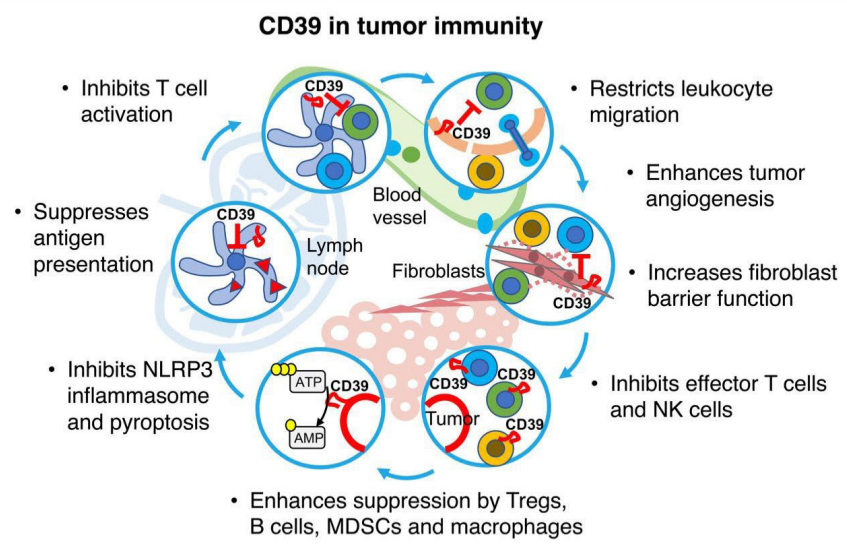

Figure 1 CD39 in the tumor immunity cycle. CD39 expression in tumors, lymph nodes and vasculature impacts tumor immunity in various intertwined ways. (1) CD39 suppresses NLRP3 inflammasome activation and pyroptosis induced by P2X7 receptor. (2) CD39 impairs immunogenic cell death and tumor antigen presentation by DCs by depleting ATP and increasing extracellular adenosine levels (3). During T cell priming, CD39 inhibits costimulatory ATP signals and increases adenosine-mediated immunosuppression. (4) CD39 expression on endothelial cells regulates transendothelial migration of immune cells and promotes angiogenesis. (5) CD39 expression on stromal cells favors fibroblasts barrier function and immunosuppression. (6) CD39 expression and activity is associated with dysfunctional T cells and NK cells, 'M2'-like macrophages and promotes immunosuppression by $T$ regulatory cells (Tregs), myeloid cells and B cell-derived microvesicles. DCs, dendritic cells; MDSCs, myeloid-derived suppressor cells; NK, natural killer.

extracellular adenosine levels, CD39 is uniquely positioned in regulation of purinergic signaling. Further in support of a critical role for CD39 in immune regulation, genome-wide association studies revealed that genetic variants of CD39 are the strongest predictor for immune-phenotypic traits, surpassing most conventional immune-related biomarkers. ${ }^{130}$ In the past recent years, highly potent mAbs targeting CD39 have been developed and were recently demonstrated to significantly reduce tumor growth in preclinical cancer models, including as single agent. Inhibiting CD39 in mice potently synergizes with other anticancer strategies, including immunotherapies, targeted therapies and chemotherapy. Given that regulators of the adenosinergic pathways display both redundant and non-redundant functions, it will be most interesting to see whether targeting CD39 can synergize with A2A receptor antagonists or CD73 inhibitors, as was observed in preclinical studies testing anti-CD73 $\mathrm{mAb}$ in combination with A2A blockade..$^{20}$ Oxygen and oxygenation agents, alone or in combination with CD39 or CD73 inhibitors, to further decrease tumor adenosine levels also constitutes another potential strategy. ${ }^{131}$ Finally, it remains unclear whether targeting of individual adenosine-generating enzymes may be limited with potential redundancies. For instance, the ecto-enzyme
CD38 can generate AMP from $\mathrm{NAD}^{+}$and has been shown to participate in tumor immune escape. ${ }^{132}$ Similarly, tissue-specific and non-specific alkalines phosphates may provide a source of extracellular adenosine independently of CD73. In summary, because of its upregulated expression levels within tumors of different types and broad immune regulatory effects on the tumor immunity cycle (figure 1), targeting CD39 currently constitutes one of the most promising approaches in immuno-oncology.

Correction notice This article has been corrected since it was published Online First. There was an error in the formatting of the P2X receptors in the text, which has since been corrected.

\section{Contributors DA, BA and JS wrote the review.}

Funding JS is supported by the Sabourin Research Chair in pharmacology and by research grants from CIHR, Canadian Cancer Society and the Terry Fox Research Institutes. DA is supported by a doctoral scholarship from the Fond de Recherche Santé - Québec.

Competing interests JS is a permanent member of the scientific advisory board of Surface Oncology, holds stocks from Surface Oncology and receives research funding from Surface Oncology.

Patient consent for publication Not required.

Provenance and peer review Not commissioned; internally peer reviewed.

Open access This is an open access article distributed in accordance with the Creative Commons Attribution Non Commercial (CC BY-NC 4.0) license, which permits others to distribute, remix, adapt, build upon this work non-commercially, and license their derivative works on different terms, provided the original work is properly cited, appropriate credit is given, any changes made indicated, and the use is non-commercial. See http://creativecommons.org/licenses/by-nc/4.0/.

\section{REFERENCES}

1 Ohta A, Sitkovsky M. Role of G-protein-coupled adenosine receptors in downregulation of inflammation and protection from tissue damage. Nature 2001;414:916-20.

2 Huang S, Apasov S, Koshiba M, et al. Role of A2A extracellular adenosine receptor-mediated signaling in adenosinemediated inhibition of T-cell activation and expansion. Blood 1997;90:1600-10.

3 Wolberg G, Zimmerman TP, Hiemstra K, et al. Adenosine inhibition of lymphocyte-mediated cytolysis: possible role of cyclic adenosine monophosphate. Science 1975;187:957-9.

4 Ohta A, Gorelik E, Prasad SJ, et al. A2A adenosine receptor protects tumors from antitumor T cells. Proc Natl Acad Sci U S A 2006;103:13132-7.

5 Takenaka MC, Gabriely G, Rothhammer V, et al. Control of tumorassociated macrophages and T cells in glioblastoma via AHR and CD39. Nat Neurosci 2019;22:729-40.

6 Allard B, Longhi MS, Robson SC, et al. The ectonucleotidases CD39 and CD73: novel checkpoint inhibitor targets. Immunol Rev 2017;276:121-44.

7 Bastid J, Regairaz A, Bonnefoy N, et al. Inhibition of CD39 enzymatic function at the surface of tumor cells alleviates their immunosuppressive activity. Cancer Immunol Res 2015;3:254-65.

8 Hayes GM, Cairns B, Levashova Z, et al. CD39 is a promising therapeutic antibody target for the treatment of soft tissue sarcoma. Am J Trans/ Res 2015;7:1181-8.

9 Pulte D, Furman RR, Broekman MJ, et al. CD39 expression on T lymphocytes correlates with severity of disease in patients with chronic lymphocytic leukemia. Clin Lymphoma Myeloma Leuk 2011;11:367-72.

10 Häusler SFM, Montalbán del Barrio I, Strohschein J, et al. Ectonucleotidases CD39 and CD73 on OVCA cells are potent adenosine-generating enzymes responsible for adenosine receptor $2 \mathrm{~A}$-dependent suppression of $\mathrm{T}$ cell function and NK cell cytotoxicity. Cancer Immunol Immunother 2011;60:1405-18.

11 Di Virgilio F, Adinolfi E. Extracellular purines, purinergic receptors and tumor growth. Oncogene 2017;36:293-303.

12 Houthuys B, Marillier R, Deregnaucourt T, et al. A novel noncompetitive and non-brain penetrant adenosine $A 2 A$ receptor 
antagonist designed to reverse adenosine-mediated suppression of anti-tumor immunity. SITC 32nd annual meeting. MA, USA, 2017.

13 Di Virgilio F, Dal Ben D, Sarti AC, et al. The P2X7 receptor in infection and inflammation. Immunity 2017;47:15-31.

14 Di Virgilio F, Sarti AC, Falzoni S, et al. Extracellular ATP and P2 purinergic signalling in the tumour microenvironment. Nat Rev Cancer 2018;18:601-18.

15 Elliott MR, Chekeni FB, Trampont PC, et al. Nucleotides released by apoptotic cells act as a find-me signal to promote phagocytic clearance. Nature 2009;461:282-6.

16 Campwala H, Sexton DW, Crossman DC, et al. P2Y(6) receptor inhibition perturbs CCL2-evoked signalling in human monocytic and peripheral blood mononuclear cells. J Cell Sci 2014;127:4964-73.

17 Schnurr M, Then F, Galambos P, et al. Extracellular ATP and TNFalpha synergize in the activation and maturation of human dendritic cells. J Immunol 2000;165:4704-9.

18 Sakaki $\mathrm{H}$, Tsukimoto $\mathrm{M}$, Harada $\mathrm{H}$, et al. Autocrine regulation of macrophage activation via exocytosis of ATP and activation of P2Y11 receptor. PLoS One 2013;8:e59778.

19 Sueyoshi K, Ledderose C, Shen Y, et al. Lipopolysaccharide suppresses T cells by generating extracellular ATP that impairs their mitochondrial function via P2Y11 receptors. J Biol Chem 2019;294:6283-93.

20 Young A, Ngiow SF, Barkauskas DS, et al. Co-Inhibition of CD73 and $\mathrm{A} 2 \mathrm{aR}$ adenosine signaling improves anti-tumor immune responses. Cancer Cell 2016;30:391-403.

21 Hatfield S, Veszeleiova K, Steingold J, et al. Mechanistic justifications of systemic therapeutic oxygenation of tumors to weaken the hypoxia inducible factor $1 \alpha$-mediated immunosuppression. Adv Exp Med Biol 2019;1136:113-21.

22 Sitkovsky MV. T regulatory cells: hypoxia-adenosinergic suppression and re-direction of the immune response. Trends Immunol 2009;30:102-8.

23 Allard B, Pommey S, Smyth MJ, et al. Targeting CD73 enhances the antitumor activity of anti-PD-1 and anti-CTLA-4 mAbs. Clin Cancer Res 2013;19:5626-35.

24 Loi S, Pommey S, Haibe-Kains B, et al. CD73 promotes anthracycline resistance and poor prognosis in triple negative breast cancer. Proc Natl Acad Sci U S A 2013;110:11091-6.

25 Young A, Ngiow SF, Madore J, et al. Targeting adenosine in BRAFmutant melanoma reduces tumor growth and metastasis. Cancer Res 2017;77:4684-96.

26 Kjaergaard J, Hatfield S, Jones G, et al. A2A2A Adenosine Receptor Gene Deletion or Synthetic A2A Antagonist Liberate Tumor-Reactive CD8+ T Cells from Tumor-Induced Immunosuppression. J Immunol 2018;201:782-91.

27 Turcotte M, Allard D, Mittal D, et al. CD73 promotes resistance to HER2/ErbB2 antibody therapy. Cancer Res 2017;77:5652-63.

28 Cekic C, Day Y-J, Sag D, et al. Myeloid expression of adenosine A2A receptor suppresses $T$ and NK cell responses in the solid tumor microenvironment. Cancer Res 2014;74:7250-9.

29 Cekic C, Linden J. Adenosine A2A receptors intrinsically regulate CD8+ T cells in the tumor microenvironment. Cancer Res 2014;74:7239-49.

30 Gaidt MM, Hornung V. The NLRP3 inflammasome renders cell death pro-inflammatory. J Mol Biol 2018;430:133-41.

31 Ben-Sasson SZ, Hogg A, Hu-Li J, et al. IL-1 enhances expansion, effector function, tissue localization, and memory response of antigen-specific CD8 T cells. J Exp Med 2013;210:491-502.

32 Segovia M, Russo S, Jeldres M, et al. Targeting TMEM176B enhances antitumor immunity and augments the efficacy of immune checkpoint blockers by Unleashing inflammasome activation. Cancer Cell 2019;35:767-81.

33 Chow MT, Sceneay J, Paget C, et al. NLRP3 suppresses NK cell-mediated responses to carcinogen-induced tumors and metastases. Cancer Res 2012;72:5721-32.

34 Molgora M, Bonavita E, Ponzetta A, et al. IL-1R8 is a checkpoint in NK cells regulating anti-tumour and anti-viral activity. Nature 2017:551:110-4.

35 Bruchard M, Mignot G, Derangère V, et al. Chemotherapy-triggered cathepsin $B$ release in myeloid-derived suppressor cells activates the NLRP3 inflammasome and promotes tumor growth. Nat Med 2013;19:57-64.

36 Voigt C, May P, Gottschlich A, et al. Cancer cells induce interleukin-22 production from memory $\mathrm{CD} 4^{+} \mathrm{T}$ cells via interleukin-1 to promote tumor growth. Proc Natl Acad Sci U S A 2017:114:12994-9.

37 Castaño Z, San Juan BP, Spiegel A, et al. IL-1ß inflammatory response driven by primary breast cancer prevents metastasisinitiating cell colonization. Nat Cell Biol 2018;20:1084-97.
38 Ridker PM, MacFadyen JG, Thuren T, et al. Effect of interleukin-1 $\beta$ inhibition with canakinumab on incident lung cancer in patients with atherosclerosis: exploratory results from a randomised, doubleblind, placebo-controlled trial. Lancet 2017;390:1833-42.

39 Ferrari D, Chiozzi P, Falzoni S, et al. Extracellular ATP triggers IL-1 beta release by activating the purinergic $\mathrm{P} 2 \mathrm{Z}$ receptor of human macrophages. J Immunol 1997;159:1451-8.

40 Wilhelm K, Ganesan J, Müller T, et al. Graft-Versus-Host disease is enhanced by extracellular ATP activating P2X7R. Nat Med 2010;16:1434-8.

41 Fuller SJ, Stokes L, Skarratt KK, et al. Genetics of the P2X7 receptor and human disease. Purinergic Signal 2009;5:257-62.

42 Gu BJ, Zhang W, Worthington RA, et al. A Glu-496 to Ala polymorphism leads to loss of function of the human $\mathrm{P} 2 \mathrm{X} 7$ receptor. J Biol Chem 2001;276:11135-42.

43 Ghiringhelli F, Apetoh L, Tesniere A, et al. Activation of the NLRP3 inflammasome in dendritic cells induces IL-1beta-dependent adaptive immunity against tumors. Nat Med 2009;15:1170-8.

44 Cabrini G, Falzoni S, Forchap SL, et al. A His-155 to Tyr polymorphism confers gain-of-function to the human $\mathrm{P} 2 \mathrm{X} 7$ receptor of human leukemic lymphocytes. J Immunol 2005;175:82-9.

45 Sun C, Chu J, Singh S, et al. Identification and characterization of a novel variant of the human $\mathrm{P} 2 \mathrm{X}(7)$ receptor resulting in gain of function. Purinergic Signal 2010;6:31-45.

46 Stokes L, Fuller SJ, Sluyter R, et al. Two haplotypes of the P2X(7) receptor containing the Ala-348 to Thr polymorphism exhibit a gain of-function effect and enhanced interleukin-1beta secretion. Faseb J 2010;24:2916-27.

47 Taylor SRJ, Gonzalez-Begne M, Dewhurst S, et al. Sequential shrinkage and swelling underlie P2X7-stimulated lymphocyte phosphatidylserine exposure and death. J Immunol 2008;180:300-8.

48 Kayagaki N, Stowe IB, Lee BL, et al. Caspase- 11 cleaves gasdermin $\mathrm{D}$ for non-canonical inflammasome signalling. Nature 2015;526:666-71.

49 Shi J, Zhao Y, Wang K, et al. Cleavage of GSDMD by inflammatory caspases determines pyroptotic cell death. Nature 2015;526:660-5

50 Kayagaki N, Warming S, Lamkanfi M, et al. Non-canonical inflammasome activation targets caspase-11. Nature 2011;479:117-21.

51 Carty M, Kearney J, Shanahan KA, et al. Cell survival and cytokine release after inflammasome activation is regulated by the Toll-IL-1R protein SARM. Immunity 2019;50:1412-24.

52 Kuhny M, Hochdörfer T, Ayata CK, et al. CD39 is a negative regulator of P2X7-mediated inflammatory cell death in mast cells. Cell Commun Signal 2014:12:40.

53 Kroemer G, Galluzzi L, Kepp O, et al. Immunogenic cell death in cancer therapy. Annu Rev Immunol 2013;31:51-72.

54 Michaud M, Martins I, Sukkurwala AQ, et al. Autophagy-dependent anticancer immune responses induced by chemotherapeutic agents in mice. Science 2011;334:1573-7.

55 Ma Y, Adjemian S, Mattarollo SR, et al. Anticancer chemotherapyinduced intratumoral recruitment and differentiation of antigenpresenting cells. Immunity 2013;38:729-41.

56 Rao S, Tortola L, Perlot T, et al. A dual role for autophagy in a murine model of lung cancer. Nat Commun 2014:5:3056.

57 Wilkin F, Duhant X, Bruyns C, et al. The P2Y11 receptor mediates the ATP-induced maturation of human monocyte-derived dendritic cells. J Immunol 2001;166:7172-7.

58 Schnurr M, Toy T, Shin A, et al. Role of adenosine receptors in regulating chemotaxis and cytokine production of plasmacytoid dendritic cells. Blood 2004:103:1391-7.

59 Yoshida O, Kimura S, Jackson EK, et al. CD39 expression by hepatic myeloid dendritic cells attenuates inflammation in liver transplant ischemia-reperfusion injury in mice. Hepatology 2013;58:2163-75

60 la Sala A, Ferrari D, Corinti S, et al. Extracellular ATP induces a distorted maturation of dendritic cells and inhibits their capacity to initiate Th1 responses. J Immunol 2001;166:1611-7.

61 Cohen HB, Briggs KT, Marino JP, et al. TLR stimulation initiates a CD39-based autoregulatory mechanism that limits macrophage inflammatory responses. Blood 2013;122:1935-45.

62 Cohen HB, Ward A, Hamidzadeh K, et al. IFN- $\gamma$ Prevents Adenosine Receptor (A2bR) Upregulation To Sustain the Macrophage Activation Response. J Immunol 2015;195:3828-37.

63 Savio LEB, de Andrade Mello P, Figliuolo VR, et al. CD39 limits P2X7 receptor inflammatory signaling and attenuates sepsisinduced liver injury. J Hepatol 2017;67:716-26.

64 Kronlage M, Song J, Sorokin L, et al. Autocrine purinergic receptor signaling is essential for macrophage chemotaxis. Sci Signal 2010;3:ra55. 
65 Chen $\mathrm{Y}$, Corriden R, Inoue $\mathrm{Y}$, et al. ATP release guides neutrophil chemotaxis via $\mathrm{P} 2 \mathrm{Y} 2$ and $\mathrm{A} 3$ receptors. Science 2006;314:1792-5.

66 Zumerle S, Calì B, Munari F, et al. Intercellular calcium signaling induced by ATP potentiates macrophage phagocytosis. Cell Rep 2019;27:1-10.

67 Feske S, Wulff $\mathrm{H}$, Skolnik EY. Ion channels in innate and adaptive immunity. Annu Rev Immunol 2015;33:291-353.

68 MacKenzie A, Wilson HL, Kiss-Toth E, et al. Rapid secretion of interleukin- 1 beta by microvesicle shedding. Immunity 2001;15:825-35.

69 Thomas LM, Salter RD. Activation of macrophages by P2X7induced microvesicles from myeloid cells is mediated by phospholipids and is partially dependent on TLR4. J Immunol 2010;185:3740-9.

70 Soni S, O'Dea KP, Tan YY, et al. ATP redirects cytokine trafficking and promotes novel membrane TNF signaling via microvesicles. Faseb J 2019;33:6442-55.

71 Kukulski F, Bahrami F, Ben Yebdri F, et al. NTPDase1 controls IL-8 production by human neutrophils. J Immunol 2011;187:644-53.

72 Reutershan J, Vollmer I, Stark S, et al. Adenosine and inflammation: CD39 and CD73 are critical mediators in LPS-induced PMN trafficking into the lungs. Faseb $J$ 2009;23:473-82.

73 Wang X, Qin W, Xu X, et al. Endotoxin-Induced autocrine ATP signaling inhibits neutrophil chemotaxis through enhancing myosin light chain phosphorylation. Proc Natl Acad Sci U S A 2017:114:4483-8.

74 Friedman DJ, Künzli BM, A-Rahim YI, et al. From the cover: CD39 deletion exacerbates experimental murine colitis and human polymorphisms increase susceptibility to inflammatory bowel disease. Proc Natl Acad Sci U S A 2009;106:16788-93.

75 Fang F, Yu M, Cavanagh MM, et al. Expression of CD39 on activated T cells impairs their survival in older individuals. Cell Rep 2016:14:1218-31.

76 Simoni Y, Becht E, Fehlings M, et al. Bystander CD $8^{+} \mathrm{T}$ cells are abundant and phenotypically distinct in human tumour infiltrates. Nature 2018:557:575-9.

77 Canale FP, Ramello MC, Núñez N, et al. CD39 Expression Defines Cell Exhaustion in Tumor-Infiltrating CD8 ${ }^{+} \mathrm{T}$ Cells. Cancer Res 2018;78:115-28.

78 Duhen T, Duhen R, Montler R, et al. Co-Expression of CD39 and CD103 identifies tumor-reactive CD8 T cells in human solid tumors. Nat Commun 2018;9:2724.

79 Thelen M, Lechner A, Wennhold K, et al. CD39 Expression Defines Cell Exhaustion in Tumor-Infiltrating $\mathrm{CD}^{+} \mathrm{T}$ Cells-Letter. Cancer Res 2018;78:5173-4.

80 Ledderose C, Liu K, Kondo Y, et al. Purinergic P2X4 receptors and mitochondrial ATP production regulate T cell migration. $J$ Clin Invest 2018:128:3583-94

81 Trimarchi H, Canzonieri R, Muryan A, et al. Podocyturia: a clue for the rational use of amiloride in Alport renal disease. Case Rep Nephrol 2016;2016:1-4.

82 Chalmin F, Mignot G, Bruchard M, et al. Stat3 and Gfi-1 transcription factors control Th17 cell immunosuppressive activity via the regulation of ectonucleotidase expression. Immunity 2012;36:362-73.

83 Ma Y, Aymeric L, Locher C, et al. Contribution of IL-17-producing gamma delta $T$ cells to the efficacy of anticancer chemotherapy. $J$ Exp Med 2011;208:491-503.

$84 \mathrm{Hu}$ X, Liu X, Moisan J, et al. Synthetic RORy agonists regulate multiple pathways to enhance antitumor immunity. Oncoimmunology 2016;5:e1254854.

85 Dulos J, Carven GJ, van Boxtel SJ, et al. Pd-1 blockade augments Th1 and Th17 and suppresses Th2 responses in peripheral blood from patients with prostate and advanced melanoma cancer. $J$ Immunother 2012;35:169-78.

86 Esfahani K, Miller WH. Reversal of autoimmune toxicity and loss of tumor response by interleukin-17 blockade. N Engl J Med 2017;376:1989-91.

87 Zhang H, Vijayan D, Li X-Y, et al. The role of NK cells and CD39 in the immunological control of tumor metastases. Oncoimmunology 2019;8:e1593809.

88 Li J, Wang L, Chen X, et al. CD39/CD73 upregulation on myeloidderived suppressor cells via TGF- $\beta$-mTOR-HIF-1 signaling in patients with non-small cell lung cancer. Oncoimmunology 2017;6:e1320011

89 Limagne E, Euvrard R, Thibaudin M, et al. Accumulation of MDSC and Th17 cells in patients with metastatic colorectal cancer predicts the efficacy of a FOLFOX-Bevacizumab drug treatment regimen. Cancer Res 2016;76:5241-52.
90 Muller L, Mitsuhashi M, Simms P, et al. Tumor-Derived exosomes regulate expression of immune function-related genes in human $T$ cell subsets. Sci Rep 2016;6:20254

91 Clayton A, Al-Taei S, Webber J, et al. Cancer exosomes express CD39 and CD73, which suppress T cells through adenosine production. J Immunol 2011;187:676-83.

92 Salimu J, Webber J, Gurney M, et al. Dominant immunosuppression of dendritic cell function by prostate-cancer-derived exosomes. $J$ Extracell Vesicles 2017:6:1368823.

93 Morandi F, Marimpietri D, Horenstein AL, et al. Microvesicles released from multiple myeloma cells are equipped with ectoenzymes belonging to canonical and non-canonical adenosinergic pathways and produce adenosine from ATP and NAD. Oncoimmunology 2018;7:e1458809.

94 Theodoraki M-N, Hoffmann TK, Jackson EK, et al. Exosomes in HNSCC plasma as surrogate markers of tumour progression and immune competence. Clin Exp Immunol 2018;194:67-78.

95 Morandi F, Marimpietri D, Horenstein AL, et al. Microvesicles expressing adenosinergic ectoenzymes and their potential role in modulating bone marrow infiltration by neuroblastoma cells. Oncoimmunology 2019;8:e1574198.

96 Zhang F, Li R, Yang Y, et al. Specific Decrease in B-Cell-Derived Extracellular Vesicles Enhances Post-Chemotherapeutic CD8 ${ }^{+} \mathrm{T}$ Cell Responses. Immunity 2019;50:738-50.

97 Plitas G, Rudensky AY. Regulatory T cells: differentiation and function. Cancer Immunol Res 2016;4:721-5.

98 Deaglio S, Dwyer KM, Gao W, et al. Adenosine generation catalyzed by CD39 and CD73 expressed on regulatory T cells mediates immune suppression. J Exp Med 2007;204:1257-65.

99 Sun X, Wu Y, Gao W, et al. CD39/ENTPD1 expression by CD4+Foxp3+ regulatory T cells promotes hepatic metastatic tumor growth in mice. Gastroenterology 2010;139:1030-40.

100 Ahlmanner F, Sundström P, Akeus P, et al. CD39 ${ }^{+}$regulatory T cells accumulate in colon adenocarcinomas and display markers of increased suppressive function. Oncotarget 2018;9:36993-7007.

101 Retseck J, Nasr A, Lin Y, et al. Long term impact of CTLA4 blockade immunotherapy on regulatory and effector immune responses in patients with melanoma. J Transl Med 2018;16:184.

102 Peres RS, Donate PB, Talbot J, et al. TGF- $\beta$ signalling defect is linked to low CD39 expression on regulatory T cells and methotrexate resistance in rheumatoid arthritis. $J$ Autoimmun 2018:90:49-58.

103 Gavin MA, Rasmussen JP, Fontenot JD, et al. Foxp3-dependent programme of regulatory T-cell differentiation. Nature 2007;445:771-5.

104 Kashyap AS, Thelemann T, Klar R, et al. Antisense oligonucleotide targeting CD39 improves anti-tumor T cell immunity. J Immunother Cancer 2019;7:67.

105 De Marchi E, Orioli E, Pegoraro A, et al. The P2X7 receptor modulates immune cells infiltration, ectonucleotidases expression and extracellular ATP levels in the tumor microenvironment. Oncogene 2019;38:3636-50.

106 Maj T, Wang W, Crespo J, et al. Oxidative stress controls regulatory T cell apoptosis and suppressor activity and PD-L1-blockade resistance in tumor. Nat Immunol 2017;18:1332-41.

107 Apetoh L, Quintana FJ, Pot C, et al. The aryl hydrocarbon receptor interacts with c-Maf to promote the differentiation of type 1 regulatory T cells induced by IL-27. Nat Immunol 2010;11:854-61.

108 Mascanfroni ID, Yeste A, Vieira SM, et al. II-27 acts on DCs to suppress the T cell response and autoimmunity by inducing expression of the immunoregulatory molecule CD39. Nat Immunol 2013;14:1054-63

109 Bergmann C, Strauss L, Zeidler R, et al. Expansion and characteristics of human T regulatory type 1 cells in co-cultures simulating tumor microenvironment. Cancer Immunol Immunother 2007;56:1429-42.

110 Mandapathil M, Szczepanski M, Harasymczuk M, et al. CD26 expression and adenosine deaminase activity in regulatory T cells (Treg) and CD4(+) T effector cells in patients with head and neck squamous cell carcinoma. Oncoimmunology 2012;1:659-69.

111 Mandapathil M, Szczepanski MJ, Szajnik M, et al. Increased ectonucleotidase expression and activity in regulatory $T$ cells of patients with head and neck cancer. Clin Cancer Res 2009;15:6348-57.

112 Jackson SW, Hoshi T, Wu Y, et al. Disordered purinergic signaling inhibits pathological angiogenesis in cd39/Entpd1-null mice. Am J Pathol 2007;171:1395-404.

113 Allard B, Turcotte M, Spring K, et al. Anti-CD73 therapy impairs tumor angiogenesis. Int J Cancer 2014;134:1466-73. 
114 Künzli BM, Nuhn P, Enjyoji K, et al. Disordered pancreatic inflammatory responses and inhibition of fibrosis in CD39-null mice. Gastroenterology 2008;134:292-305.

115 Fernández P, Perez-Aso M, Smith G, et al. Extracellular generation of adenosine by the ectonucleotidases CD39 and CD73 promotes dermal fibrosis. Am J Pathol 2013;183:1740-6.

116 Fernández P, Trzaska S, Wilder T, et al. Pharmacological blockade of $A 2 A$ receptors prevents dermal fibrosis in a model of elevated tissue adenosine. Am J Pathol 2008;172:1675-82.

117 Perera LMB, Sekiguchi A, Uchiyama A, et al. The Regulation of Skin Fibrosis in Systemic Sclerosis by Extracellular ATP via P2Y Purinergic Receptor. J Invest Dermatol 2019;139:890-9.

118 Dranoff JA, Ogawa M, Kruglov EA, et al. Expression of P2Y nucleotide receptors and ectonucleotidases in quiescent and activated rat hepatic stellate cells. Am J Physiol Gastrointest Liver Physiol 2004;287:G417-24.

119 Gonçalves RG, Gabrich L, Rosário A, et al. The role of purinergic $\mathrm{P} 2 \mathrm{X} 7$ receptors in the inflammation and fibrosis of unilateral ureteral obstruction in mice. Kidney Int 2006;70:1599-606.

120 Lu D, Soleymani S, Madakshire R, et al. Atp released from cardiac fibroblasts via connexin hemichannels activates profibrotic P2Y2 receptors. Faseb J 2012;26:2580-91.

121 Riteau N, Gasse P, Fauconnier L, et al. Extracellular ATP is a danger signal activating $\mathrm{P} 2 \mathrm{X} 7$ receptor in lung inflammation and fibrosis. Am J Respir Crit Care Med 2010;182:774-83.

122 Feng L, Sun X, Csizmadia E, et al. Vascular CD39/ENTPD1 directly promotes tumor cell growth by scavenging extracellular adenosine triphosphate. Neoplasia 2011;13:206-IN2.
123 Künzli BM, Bernlochner M-I, Rath S, et al. Impact of CD39 and purinergic signalling on the growth and metastasis of colorectal cancer. Purinergic Signal 2011;7:231-41.

124 Perrot I, Michaud H-A, Giraudon-Paoli M, et al. Blocking antibodies targeting the CD39/CD73 immunosuppressive pathway Unleash immune responses in combination cancer therapies. Cell Rep 2019;27:2411-25.

125 Yadav V, Chi L, Zhao R, et al. Ectonucleotidase tri(di) phosphohydrolase-1 (ENTPD-1) disrupts inflammasome/interleukin 1ß-driven venous thrombosis. J Clin Invest 2019;129:2872-2877.

126 Kanthi Y, Hyman MC, Liao H, et al. Flow-dependent expression of ectonucleotide tri(di)phosphohydrolase-1 and suppression of atherosclerosis. J Clin Invest 2015;125:3027-36.

127 De Giorgi M, Enjyoji K, Jiang G, et al. Complete deletion of Cd39 is atheroprotective in apolipoprotein E-deficient mice. J Lipid Res 2017;58:1292-305.

128 Cho MS, Noh K, Haemmerle M, et al. Role of ADP receptors on platelets in the growth of ovarian cancer. Blood 2017;130:1235-42.

129 Enjyoji K, Kotani K, Thukral C, et al. Deletion of cd39/entpd1 results in hepatic insulin resistance. Diabetes 2008;57:2311-20.

130 Orrù V, Steri M, Sole G, et al. Genetic variants regulating immune cell levels in health and disease. Cell 2013;155:242-56.

131 Hatfield SM, Kjaergaard J, Lukashev D, et al. Immunological mechanisms of the antitumor effects of supplemental oxygenation. Sci Transl Med 2015;7:277ra30.

132 Chen L, Diao L, Yang Y, et al. Cd38-Mediated immunosuppression as a mechanism of tumor cell escape from PD-1/PD-L1 blockade. Cancer Discov 2018;8:1156-75. 